\title{
Tobacco Use Policy Research
}

National Cancer Institute

\section{Source}

National Cancer Institute. Tobacco Use Policy Research. NCI Thesaurus. Code C15993.

Research studies that assess the effect of policies, including worksite, community, state and Federal policies, on tobacco initiation and use. (NCI/T RIP) 